\title{
Micrometer-sized water ice particles for planetary science experiments: influence of surface structure on collisional properties
}

\author{
S. Gärtner, B. Gundlach, T. F. Headen, J. Ratte, J. Oesert, \\ S. N. Gorb, T. G. A. Youngs, D. T. Bowron, \\ J. Blum, and H. J. Fraser
}

\section{Published version information}

Citation: S Gärtner et al. "Micrometer-sized water ice particles for planetary science experiments: influence of surface structure on collisional properties." Astrophysical Journal, vol. 848, no. 2 (2017): 96.

DOI: $\underline{10.3847 / 1538-4357 / a a 8 c 7 f}$

This version is made available in accordance with publisher policies. Please cite only the published version using the reference above. This is the citation assigned by the publisher at the time of issuing the APV. Please check the publisher's website for any updates. 


\title{
Micrometer-sized Water Ice Particles for Planetary Science Experiments: Influence of Surface Structure on Collisional Properties
}

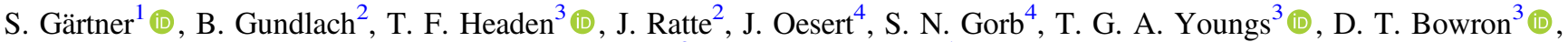 \\ J. Blum ${ }^{2}$ (D), and H. J. Fraser ${ }^{1}$ (D) \\ ${ }^{1}$ School of Physical Sciences, The Open University, Walton Hall, Milton Keynes MK7 6AA, UK; sabrina.gaertner@stfc.ac.uk, helen.fraser@open.ac.uk \\ ${ }^{2}$ Institut für Geophysik und extraterrestrische Physik, TU Braunschweig, Mendelssohnstr. 3, D-38106 Braunschweig, Germany \\ ${ }^{3}$ ISIS Facility, STFC Rutherford Appleton Laboratory, Harwell Oxford, Didcot OX11 0QX, UK \\ ${ }^{4}$ Zoologisches Institut, Christian-Albrechts-Universität zu Kiel, Am Botanischen Garten 1-9, D-24118 Kiel, Germany \\ Received 2017 July 31; revised 2017 September 11; accepted 2017 September 11; published 2017 October 17
}

\begin{abstract}
Models and observations suggest that ice-particle aggregation at and beyond the snowline dominates the earliest stages of planet formation, which therefore is subject to many laboratory studies. However, the pressuretemperature gradients in protoplanetary disks mean that the ices are constantly processed, undergoing phase changes between different solid phases and the gas phase. Open questions remain as to whether the properties of the icy particles themselves dictate collision outcomes and therefore how effectively collision experiments reproduce conditions in protoplanetary environments. Previous experiments often yielded apparently contradictory results on collision outcomes, only agreeing in a temperature dependence setting in above $\approx 210 \mathrm{~K}$. By exploiting the unique capabilities of the NIMROD neutron scattering instrument, we characterized the bulk and surface structure of icy particles used in collision experiments, and studied how these structures alter as a function of temperature at a constant pressure of around $30 \mathrm{mbar}$. Our icy grains, formed under liquid nitrogen, undergo changes in the crystalline ice-phase, sublimation, sintering and surface pre-melting as they are heated from 103 to $247 \mathrm{~K}$. An increase in the thickness of the diffuse surface layer from $\approx 10$ to $\approx 30 \AA$ ( $\approx 2.5$ to 12 bilayers) proves increased molecular mobility at temperatures above $\approx 210 \mathrm{~K}$. Because none of the other changes tie-in with the temperature trends in collisional outcomes, we conclude that the surface pre-melting phenomenon plays a key role in collision experiments at these temperatures. Consequently, the pressure-temperature environment, may have a larger influence on collision outcomes than previously thought.
\end{abstract}

Key words: accretion, accretion disks - methods: laboratory: solid state - planets and satellites: formation

\section{Introduction}

Dust aggregation is a key step in planet formation (Blum \& Wurm 2008; Wada et al. 2008; Garaud et al. 2013; Testi et al. 2014), enhanced by water ice at and beyond the snowline (Aumatell \& Wurm 2011; Kataoka et al. 2013; Gundlach \& Blum 2015). However, we can only quantify collisional outcomes empirically to learn how icy dust sticks under protoplanetary conditions (e.g., Bridges et al. 1996; Higa et al. 1996; Gundlach \& Blum 2015; Hill et al. 2015).

Protoplanetary disk models indicate that icy particles are continually processed as particles traverse temperature and density gradients (Visser et al. 2009; Woitke 2015; Woitke et al. 2016), resulting in repeated evaporation and reformation of the water ice, which may be amorphous, crystalline, or a mixture of both (Sirono 2011a, 2011b; Ros \& Johansen 2013; Sirono \& Ueno 2017). Indeed, both types have been observed in accretion disks (Schegerer \& Wolf 2010; Terada \& Tokunaga 2012; Boogert et al. 2015). Previous experiments (Wang et al. 2005) showed that crystalline ice films absorb much less energy from impacts than amorphous ices, implying that collisional outcomes between protoplanetary disk particles could vary as a function of the ice phase.

However, all laboratory experiments necessarily have to use analogs rather than interstellar ice, and the ice-particle formation mechanisms in the laboratory diverge from those in astronomical environments. We therefore address the following outstanding questions.
1. Are the icy particles we are colliding in laboratory experiments good analogs for protoplanetary disk environments?

2. Does the ice phase of our particles affect the collisional outcome?

3. Does the surface structure play a dominant role?

We have exploited neutron scattering and cryo-SEM (scanning electron microscopy) to characterize the ice-particle analogs used in our laboratory collision experiments (Gundlach \& Blum 2015) to ascertain whether phase changes in the bulk ice, and/or surface structural changes, tie-in with the temperature dependencies observed in collisional data, and whether the production method of the ice analogs influences the particle structure.

In combination, these data reveal which of the ice properties can affect collisional outcomes and to what extent these properties are altered by the collision environment. This information is essential to relate laboratory data back to planet formation scenarios and to disentangle the seemingly contradictory results from laboratory collision experiments performed under different conditions, such that the most appropriate data can be employed in planet forming models, and where necessary such models can be modified to account for the influence of ice physics on collision outcomes.

\section{Outstanding Challenges from Empirical Ice Collision Data}

Generally, planet formation requires aggregation of small particles to form bigger ones. However, particle sticking 
(a perquisite of models) is observed only in a small subset of collision experiments and then over a range of sticking probabilities (20\%-100\%; Hatzes et al. 1991; Bridges et al. 1996; Shimaki \& Arakawa 2012b; Gundlach \& Blum 2015; Deckers \& Teiser 2016; Musiolik et al. 2016). Interestingly, all of these studies were performed at relatively high pressures $\left(1-10^{3}\right.$ mbar), so that we cannot know whether the results would have been the same at lower pressures as expected beyond the snowline of protoplanetary disks $(<1 \mathrm{mbar}$; Cieza et al. 2016).

All laboratory experiments where sticking is observed are similar in that they involved micrometer-sized structures (small particles or layers of condensed water often referred to as "frost"). Indeed, models predict that the particle size strongly influences the sticking probability during collisions: the sticking threshold velocity $v_{\text {stick }}$ decreases with increasing particle radius $r: v_{\text {stick }} \propto r^{-2 / 3}$ for $0.1-10 \mu \mathrm{m}$ sized particles (see Figure 12 in Gundlach \& Blum 2015), and $v_{\text {stick }} \propto r^{-1}$ for millimeter- to meter-sized particles (see Figure 7.1 in Heißelmann 2015). However, micrometer-sized features on the surface of centimeter-sized particles (as induced by roughening or water condensation) are far less predictable, and several collisional studies on such particles did not observe any sticking (Hatzes et al. 1988; McDonald et al. 1989; Dilley \& Crawford 1996; Higa et al. 1996).

In collision experiments that do not lead to sticking, the coefficient of restitution, $\epsilon$, is extracted, which describes the loss of translational energy resulting from the collision, and eventually feeds into models of planet formation. However, previous experiments (Bridges et al. 1984; Hatzes et al. 1988; McDonald et al. 1989; Hatzes et al. 1991; Supulver et al. 1995; Dilley \& Crawford 1996; Bridges et al. 1996; Higa et al. 1996, 1998; Heißelmann et al. 2010; Shimaki \& Arakawa 2012a, 2012b; Gundlach \& Blum 2015; Hill et al. 2015; Deckers \& Teiser 2016; Musiolik et al. 2016) disagree on whether, and how, $\epsilon$ varies as a function of temperature, pressure, velocity, size, and shape. The question is, why is this? We hypothesize that two key factors play a role: first the method and prevailing conditions under which the icy particles are formed, and second the prevailing conditions under which the particle collisions are investigated.

The two key environmental parameters are pressure $P$ and temperature $T$. However, these two parameters are not usually varied systematically, resulting in contradictory experimental results and making it difficult to ascertain exactly which empirical data are most relevant to planet-forming models. From the few cases where $T$ has been varied at constant $P$ (Bridges et al. 1984; McDonald et al. 1989; Higa et al. 1996; Heißelmann et al. 2010; Gundlach \& Blum 2015; Hill et al. 2015), two clear trends are evident; the collisional outcomes are temperature-independent below $T \approx 210 \mathrm{~K}$ (e.g., Heißelmann et al. 2010; Gundlach \& Blum 2015; Hill et al. 2015) and become temperature-dependent above $T \approx 210 \mathrm{~K}$ (e.g., Gundlach \& Blum 2015), where the coefficient of restitution decreases and the threshold velocity to particle sticking increases, as temperature increases.

The ice projectiles in these collision experiments have been formed under various conditions, but always from the liquid phase. While freezing of liquid water in a kitchen freezer or under liquid nitrogen is expected to yield some form of crystalline ice, the ice structure on a molecular scale will depend on the freezing rate as well as on the conditions (and duration) under which the ice was processed and/or stored between initial freezing and eventual collision. This "thermal history" again has not always been varied systematically and has not even always been fully described.

Micrometer-sized particles can be created by shattering larger bodies of ice prepared in a freezer (Deckers \& Teiser 2016), or by rapid freezing of water droplets, e.g., on cold surfaces (Musiolik et al. 2016) or by introducing them into cold gaseous or liquid environments (Shimaki \& Arakawa 2012b; Gundlach \& Blum 2015), However, without further characterization, we cannot know whether, and how, the production alters the particle structure on all length scales, exactly which form of crystalline ice is produced, and to what extend the $P-T$ conditions of the collision environment influence the collisional outcomes.

Because no definitive particle characterizations have been made to date, ice phase and micro-scale structure are alluded to in icy particle collision experiments and their influence on collision outcomes remains a contentious issue in the literature, which we address in this work.

By exploiting the unique capabilities of the NIMROD (Bowron et al. 2010) neutron scattering instrument, we characterized the bulk and surface structure of the icy particles. NIMROD can simultaneously observe a wide range of length scales from the mesoscale $(\approx 60 \mathrm{~nm})$ down to the intramolecular level, which means it is possible to concurrently establish the phase, molecular structure, and surface properties of icy materials (Mitterdorfer et al. 2014; Hill et al. 2016).

\section{Experimental Method}

The particles for this characterization study were produced as described in detail in Gundlach et al. 2011, Jost et al. 2013, and Gundlach \& Blum 2015. Briefly, liquid $\mathrm{D}_{2} \mathrm{O}$ was dispersed by an aspirator and sprayed into liquid nitrogen, accumulating sample material for $>1 \mathrm{hr}$. The particles were then funneled into the pre-cooled $(77 \mathrm{~K})$ sample container, which was closed, mounted in the neutron beam, and passively cooled at a constant $P$ (30 mbar He).

We studied samples with two different mean particle radii, $((0.71 \pm 0.31) \mu \mathrm{m}$ and $(1.45 \pm 0.65) \mu \mathrm{m})$, where uncertainties give the particle size distribution's full width at half maximum (FWHM; Gundlach et al. 2011). Two independent experiments were conducted at each particle radius.

Advantage was taken of the neutron scattering properties of $\mathrm{D}_{2} \mathrm{O}$ compared to $\mathrm{H}_{2} \mathrm{O}$ (Sears 1992) and care was taken to minimize sample contamination with $\mathrm{H}_{2} \mathrm{O}$ during sample preparation and loading, by maintaining an $\mathrm{N}_{2}$-purged environment, retaining both the container and sample below $100 \mathrm{~K}$.

Neutron scattering data was collected over 30-minute isothermal periods, at 103, 164, 184, 206, 226, and $247 \mathrm{~K}$. The initial data reduction and calibration was done using GudrunN software (Soper 2011, 2013), according to standard neutron scattering data processing. The raw data from our neutron scattering experiments were merged for all detectors, corrected for instrument effects, and normalized on a per atom basis. Examples of the resulting background-corrected neutron diffraction patterns are shown in Figure 1(a) for one of the four experiments. The small angle neutron scattering $\left(Q \leqslant 0.1 \AA^{-1}\right)$, probing surface structures, was observed concurrently with the high- $Q$ region $\left(Q \geqslant 1 \AA^{-1}\right)$, probing the bulk-ice phase (intramolecular distances).

To give an impression of the particle structures on larger scales $(0.1-100 \mu \mathrm{m})$, we compared the neutron scattering results to images from complementary cryo-SEM experiments 

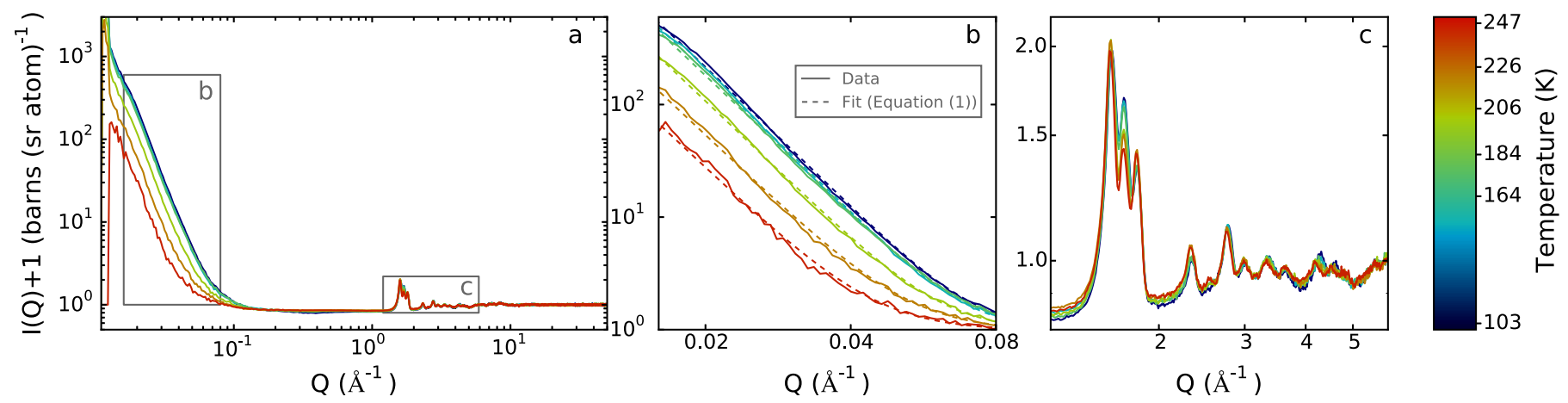

Figure 1. (a) NIMROD spectra $\left.(0.71 \mu \mathrm{m} \text { icy particles), showing the neutron scattering signal } I(Q) \text { (barns (sr atom) })^{-1}\right)$ as a function of the momentum transfer $Q$, which is inversely proportional to the length scale. Sequential plots show the temperature evolution in six isothermal steps between 103 and $247 \mathrm{~K}$ (color bar). Expanded views: (b) low- $Q$ (dashed lines show the fit as per Equation (1), Section 4.2) and (c) high- $Q$ regions. For clarity, error bars have been omitted (average uncertainty: $6 \%$ of $I(Q))$.

(Hitachi, S-4800; ${ }^{5}$ see also Jost et al. 2013), using $\mathrm{H}_{2} \mathrm{O}$-particles prepared the same way as previously described, but necessarily held at lower pressures $\left(P=10^{-3} \mathrm{mbar}\right)$.

\section{Results}

Across the four neutron scattering experiments, no clear differences were seen between scattering from different icy particle sizes, nor in repeated experiments on particles of the same size. However, clear changes with increasing sample temperature are evident (Figure 1), indicating temperature induced modifications in the icy particles, both in the bulk-ice phase and the particle surfaces, which will be addressed in detail in the following subsections.

\subsection{Ice Phase}

The high- $Q$ region (Figure 1(c)) shows peaks indicative of a crystalline-dominated ice structure; the most prominent feature is the triplet of Bragg peaks in exactly the positions expected for hexagonal ice, $I_{h}\left(1.59,1.70\right.$, and $1.80 \AA^{-1}$ Petrenko \& Whitworth 1999). There are some subtle but significant modifications to this triplet as a function of temperature; the peak at $1.70 \AA^{-1}$ actually corresponds to overlapping features from $I_{h}$ and $I_{c}$ (cubic crystalline ice) and loses intensity above $180 \mathrm{~K}$. This is also reflected in changes to the smaller diffraction peaks at higher $Q$ and indicates that $I_{c}$ is lost. There are two possible pathways for this loss; either transformation to the more stable $I_{h}$ or sublimation. Any increase in the amount of $I_{h}$ would result in an increase in the intensities of the other $I_{h}$ Bragg peaks, which appear unaltered.

To quantify these changes, we deconstructed each high- $Q$ diffraction pattern between $Q$ values of 1-6 $\AA^{-1}$ into a sum of features representing $I_{h}$ and $I_{c}$, based on crystallographic calculations of the diffraction patterns for the respective idealized pure ice phases. The residuals of this analysis showed a very broad diffraction peak, whose shape and position closely matched that expected for amorphous ice (Figure 2(a) blue and yellow curves), so a third component was added to the deconstruction; $I_{x}$, denoting inter-domain bulk material of no long-range order, which will be discussed in more detail in Section 5.3.

Within fitting uncertainties, this deconstruction showed that the temperature dependence of the ice structure is independent

\footnotetext{
5 Hitachi S-4800, Hitachi High-Technologies Corp., (Tokyo, Japan), equipped with a Gatan ALTO 2500 cryo-preparation system, Gatan Inc., (Abingdon, UK).
}

of particle radius, i.e., a bulk effect. Initially, the icy particles exhibit stacking disorder, dominated by $I_{h}$. They comprise areas of both $I_{h}$ and $I_{c}$ as well as inter-domain amorphous structures, $I_{x}$.

As $T$ increases, the normalized fractions of all three components change and the normalized fractions no longer sum to 1 . A measurable fraction of $I_{c}$ is retained until $184 \mathrm{~K}$ (Figure 2(b) regime (A)), but has essentially disappeared by $206 \mathrm{~K}$. A fraction of $I_{x}$ persists until $226 \mathrm{~K}$ (Figure 2(b) regime (B)); at $247 \mathrm{~K}$ the data is best fitted by $I_{h}$ only.

\subsection{Surface}

Returning to Figure 1(b), the low- $Q$ data show much more drastic changes than the high- $Q$ Bragg peaks. There is little, if any, obvious change in $I(Q)$ between 103 and $184 \mathrm{~K}$, but as $T$ increases beyond $184 \mathrm{~K}, I(Q)$ diminishes rapidly. This indicates a substantial change in the particles' surface structure, the onset of which coincides with the temperature regime at which $I_{c}$ is no-longer measurable in the bulk-ice.

At all temperatures, the low- $Q$ slopes approximately follow the expected Porod power law (Feigin et al. 1987), indicative of compact, granular material. This is expected from a sample of nonporous, spherical icy particles and thus confirms that formation in liquid nitrogen does not alter the internal particle structures on length scales of tens of nanometers.

There are well established methods to extract quantitative information on the surface structures from these data. Porod analysis (Sinha et al. 1988) yields a Porod exponent $\beta$, which can be related to the ice surface roughness (e.g., Mitterdorfer et al. 2014; Hill et al. 2016). For our icy particles, $\beta$ values ranged from 4.1 to 6 , increasing nonlinearly with increasing temperature. Values of $\beta>4$ indicate that no surface roughness on nanometer-length scales is introduced by the freezing process, but that the surfaces are diffuse, i.e., showing a density gradient (e.g., Strey et al. 1991; Su et al. 1998). However, in the case of diffuse interfaces, the particle surface density cannot be validly modeled by a step function (as in the Porod analysis) but is best described by convoluting a Gaussian with said step function. The width of this Gaussian indicates the thickness of the diffuse interface, $t$. The resulting fit-function for the background-corrected low- $Q$ data is (Strey et al. 1991)

$$
I(Q)=2 \pi(\Delta \rho)^{2} \mathrm{SSA} Q^{-4} e^{-Q^{2} t^{2}},
$$

where SSA is the specific surface area, and $\Delta \rho=$ $5.995 \times 10^{-6} \AA^{-1}$ is the scattering length density difference. 


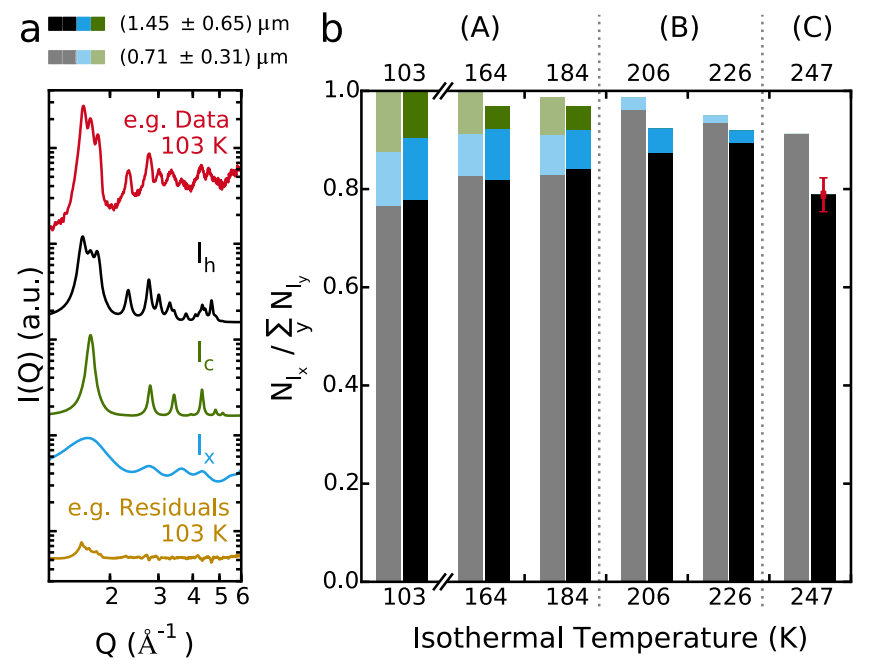

Figure 2. (a) Original high- $Q$ data for the $103 \mathrm{~K}, 0.71 \mu \mathrm{m}$ particles (red), together with diffraction patterns of the three ice phase components $I_{h}$ (black), $I_{c}$ (green), and $I_{x}$ (blue). Each high- $Q$ pattern was deconstructed into a sum of these components as described in the text. Also shown (yellow) are the residuals when only $I_{h}$ and $I_{c}$ are fitted. (b) Outcome of this analysis across our entire data set. Cumulative bars represent the fractions of each ice phase (colors are the same as in (a)) required to reproduce the high- $Q$ data at each isothermal temperature, averaged across two experimental runs, and separated by particle radius (see the legend). Each cumulative bar is normalized to the total amount of ice determined in each sample at the initial temperature point, $103 \mathrm{~K}$. The typical uncertainty, propagated from the individual fits, is shown on the final $I_{h}$ bar; for clarity, other error bars have been omitted. Dotted lines indicate the critical isothermal steps at which drastic changes in the ice phase were observed, breaking the data into three distinct ice-phase regimes; $I_{h}+I_{c}+I_{x} ; I_{h}+I_{x} ; I_{h}$.

Under our specific experimental conditions, this is the scattering length density of $\mathrm{D}_{2} \mathrm{O}$, since no other material is present that has not already been corrected for by the calibration scans. The resulting fits ideally reproduce the experimental data over the entire low- $Q$ range, (Figure 1(b): dashed lines).

A major advantage of this model is that it concurrently gives values of $t$ and SSA, (Figure 3). At all temperatures, the SSA values are below those calculated for samples of smooth spherical particles with the given size distributions (Figure 3 left-hand axis: light/dark $-\times-$ ), which will be discussed in detail in Section 5.2. However, as expected, the SSA of the $0.71 \mu \mathrm{m}$ particles is always greater than that of the $1.45 \mu \mathrm{m}$ particles. Regardless of particle size, the SSA slightly decreases in the 103-184 K range; the most drastic changes in SSA set in beyond $184 \mathrm{~K}$, then this loss rate slows beyond $226 \mathrm{~K}$.

It is interesting to note that when the regimes (dotted vertical lines) from Figure 2 are transposed to Figure 3, the key temperatures at which ice-phase-compositional changes occur correspond exactly with the distinctive changes in SSA.

Whilst changes in $t$ almost mirror those in SSA, the most drastic changes occur above $206 \mathrm{~K}$, thus not matching the temperature regimes observed for ice phase and SSA. The absolute values of $t$ are closely comparable between the two particle sizes, starting from around $10 \AA$ at $103 \mathrm{~K}$, which represents roughly $2.5 I_{h}$ bilayers, and increase (on average) by a factor of 3 with temperature.

From the SSA and $t$ results alone, we cannot distinguish whether the surface changes are caused by particle sintering or by sublimation. However, the images obtained from our complementary cryo-SEM study can answer this question.

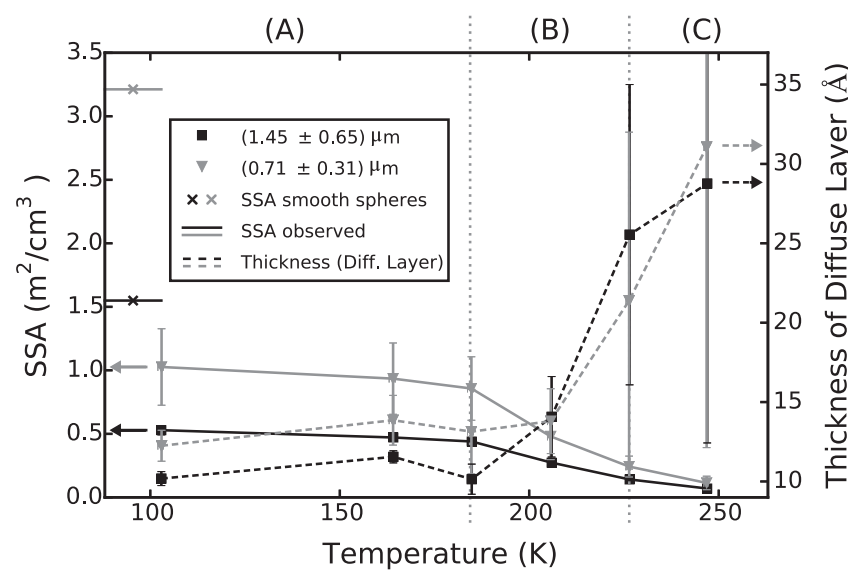

Figure 3. SSA and $t$, as a function of temperature, as extracted from the low- $Q$ NIMROD data using Equation (1). For clarity, the results have been averaged per particle size across experimental runs, and consecutive temperature points joined with a solid line (SSA, left-hand axis) or dashed line ( $t$, right-hand axis). Error bars represent the standard deviation of the mean.

\subsection{Sintering or Sublimation?}

Figure 4 joins up our findings on all length scales together with the SEM images. The vertical dotted lines indicate the same evolutionary stages in the ice-phase composition and SSA, as identified in Figures 2 and 3.

The SEM images reveal that initially the particles are mostly, although not perfectly, spherical (Figures 4(a)-(c)). The icy particles are in contact with each other, but no sintering is evident. With increasing temperature, sintering is observed (Figure $4\left(b_{2}\right)$ ), and as the temperature continues to rise, the sintering necks become more pronounced (Figure $4\left(c_{2}\right)$ ).

Finally, by the highest temperatures, where the particles only comprise $I_{h}$ (i.e., beyond the second vertical dotted line in Figures 2-4), material seems to be lost from the narrow sinterneck and the particles become faceted with straight edges and reduce in size (Figure $4\left(d_{2}\right)$ ), while the smallest particles are lost.

\section{Discussion}

We have characterized micrometer-sized icy particles identical to those used in laboratory collision experiments on planet formation. Our icy particles were produced under liquid nitrogen and were not dissimilar in size to grains of crystallized water ice $(\approx 0.8 \mu \mathrm{m}$ in size $)$ that have been observed in the silhouette disk of a young star (Terada \& Tokunaga 2012).

Our characterization experiments cover almost the whole temperature range exploited in laboratory collisional studies over the past three decades, from 80 K (e.g., Hatzes et al. 1988; Musiolik et al. 2016) to 269 K (e.g., Higa et al. 1996; Shimaki \& Arakawa 2012b), performed to understand collisions in a variety of environments, like protoplanetary disks, cometary surfaces, and planetary rings. Thus, our results provide crucial information toward the role of the ice phase and surface structures in dictating collision outcomes in such environments.

\subsection{Does the Ice Phase Affect the Collisional Outcome?}

We find that our particles are initially stacking disordered, as is expected when freezing water droplets in liquid nitrogen (Kuhs et al. 2012; Malkin et al. 2012, 2015). The particles comprise both low-pressure crystalline phases of ice, $I_{c}$ and $I_{h}$, 


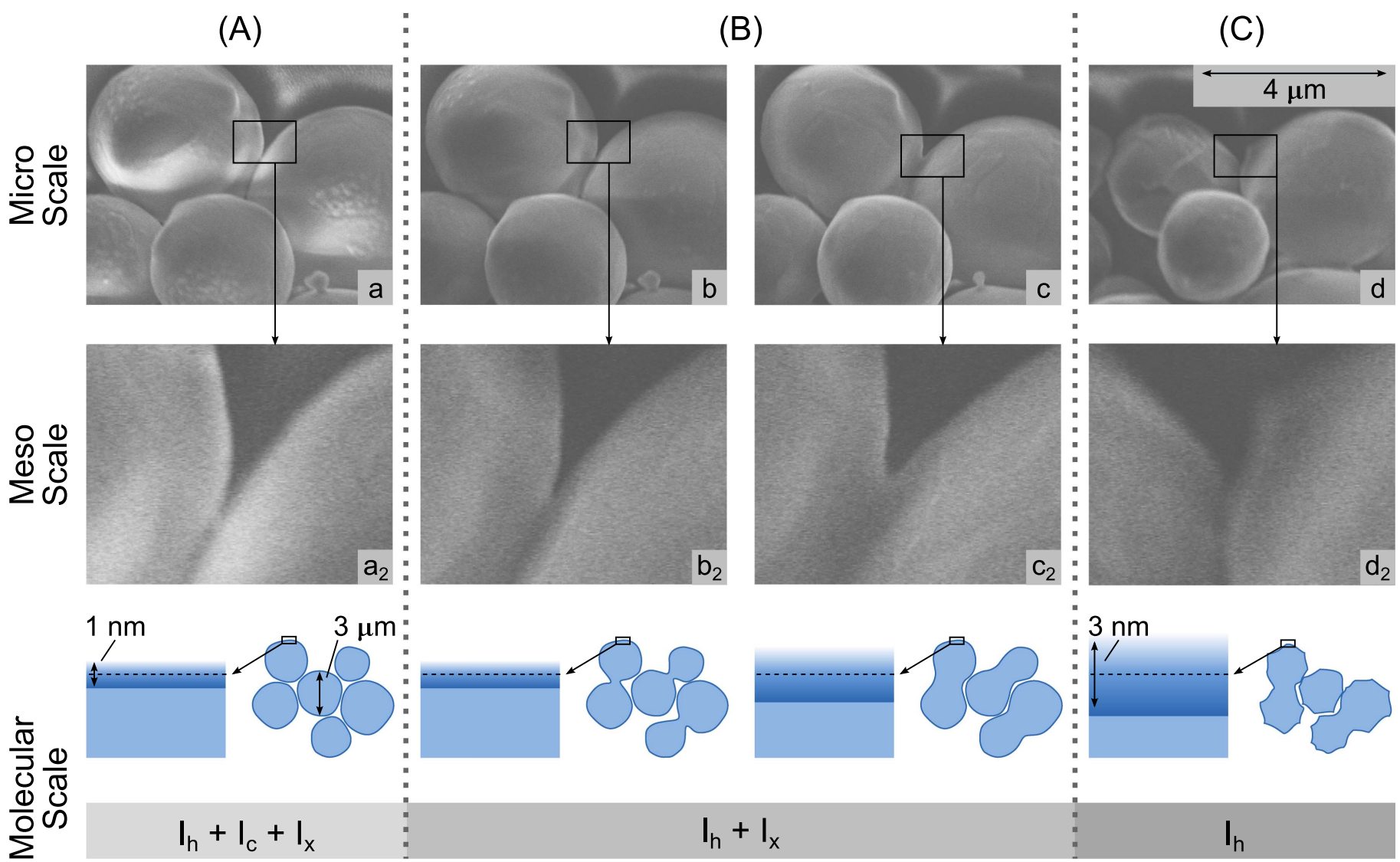

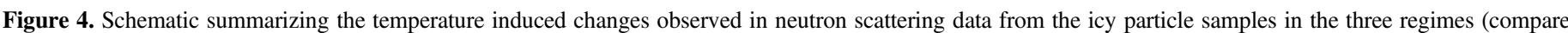

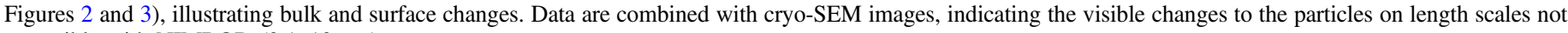
accessible with NIMROD $(0.1-10 \mu \mathrm{m})$.

but the obtained diffraction patterns are best fitted when an amorphous ice phase is added. Various amorphous ice candidates could be attributed to this third phase, e.g., hyperquenched glassy water (HGW), low-density amorphous solid water (LDA), and high-density amorphous solid water (HDA), but the resolution of our diffraction data is not well suited to distinguish between them. NIMROD was not designed as a high-resolution crystallography instrument, but rather to provide atomistically quantitative structural data for highly disordered and complex systems over a very wide $Q$-range. Sophisticated models for the fitting of diffraction patterns from stacking disordered ices have been developed by various groups (e.g., Kuhs et al. 2012; Malkin et al. 2015, and references therein). However, an in-depth analysis of the obtained diffraction patterns is not required for the purpose of this work and is not feasible using the moderate resolution data obtained.

Nevertheless, our molecular-scale neutron scattering data are sufficient to characterize three distinct phase regimes for our icy particles (Figures 2 and 4). The temperature range, across which contributions from $I_{x}$ are seen, supports the interpretation as inter-domain ice (lacking long-range order) sandwiched between hexagonal and cubic domains, as illustrated, e.g., in Figures 2 and 3 of Hondoh (2015). These inter-domain amorphous structures; are not equivalent to, nor to be confused with, diffuse surface layers or vapor-deposited amorphous solid water (ASW). We find that none of the phase-change temperatures matches the collisional temperature dependencies, which set in above $\approx 210 \mathrm{~K}$. Thus, we conclude that bulk crystalline ice-phase cannot influence collisional outcomes in our experiments and further crystallographic studies on a dedicated instrument are not required.

\subsection{Does the Surface Play a Dominant Role?}

Surface features could be connected to collision outcomes and particle aggregation in several ways. Both surface wetting and surface roughness might be expected to increase the stickiness of particles via friction effects. Molecular-scale features ( $\AA$-scale), such as molecular orientation, mobility, or density variations on the surface might affect particle stickiness. They would affect the small angle scattering slope, but not the SSA. Surface roughness on nanometer-scales would affect the small angle scattering slope and increase the observed SSA, particle sintering in the aggregation process would reduce the SSA with respect to that of smooth spherical particles.

Based on the original Porod analysis of our small angle scattering data, we can exclude surface roughness, and indeed we find that even at the lowest temperatures the observed SSA is below that expected from the given size distributions of spherical particles by a factor of $\approx 3$ for both mean particle sizes (Figure 3), which could indicate particle sintering. However, the SEM images (Figure $4\left(a_{2}\right)$ ) reveal that at the lowest temperatures the particles are mostly, but not perfectly, spherical and in contact, slightly reducing the observable SSA, although no sintering is evident yet. 
As the temperature is increased, we observe a gradual loss in SSA up to around $184 \mathrm{~K}$ (Figure 3), which is explained by the onset of particle sintering, evident in the SEM images (Figure 4 $\left.\left(b_{2}\right)\right)$. While such ice particles still show temperature independent outcomes in laboratory collision experiments (Gundlach \& Blum 2015), the sintering of particles in close proximity, even at such low temperatures and pressures, corroborates earlier suggestions (Sirono 2011b; Sirono \& Ueno 2017) that protoplanetary disk particles in contact will eventually merge together over long timescales, thereby forming conjoined objects.

Sintering becomes more pronounced with further increases in temperature (Figure $4\left(c_{2}\right)$ ) commensurate with the rapid SSA loss beyond $184 \mathrm{~K}$ (Figure 3), and the particles shrink in diameter (Figures 4(c) and (d)). This suggests a loss of ice via sublimation, which agrees with our earlier finding that the normalized fractions of ice phases (Figure 2) sum to 1 only at the lowest temperature. This is also supported by the GudrunN processing of the original data: the total scattering at high $Q$ yields volume filling factors (not shown); these indicate which fraction of the probed volume is filled with sample material and also point to the amount of sample slightly decreasing with increasing $T$.

At the highest temperatures (Figure $4\left(\mathrm{~d}_{2}\right)$ ), material is lost even from the sinter necks, commensurate with the decrease in the rate of SSA change, observed via neutron scattering.

The SSA results come with one caveat; the initial SSA is lower than could be explained by particle contact alone. Possible reasons for this are baseline errors in the corrected neutron scattering data (attributable to potential $\mathrm{H}_{2} \mathrm{O}(\leqslant 4 \%)$ contamination in the samples, affecting the absolute $I(Q)$ calibration) could introduce a systematic error (up to a factor of 1.3) to the absolute SSA values, which therefore must be considered lower limits. The size distribution derived in earlier studies might be slightly altered by a different tube length between aspirator and liquid nitrogen Dewar or by size segregation effects during the filling process. We assume that all of these affect the absolute SSA values to some degree and in combination explain the observed discrepancies.

While the absolute values of SSA might be affected by the above systematic deviations, the trends are not, and changes in the SSA are indicative of changes to the particle's surface structures, which might affect collision outcomes.

While both sintering and sublimation could affect particle stickiness, the temperature trends in the SSA and larger scale surface structures are not commensurate with those in collision experiments. Thus, they cannot play a key role in determining collision outcomes.

We therefore return to the surface features on $\AA$-scales: while the Porod analysis excluded surface roughness, it pointed to our particles having a diffuse interface at all temperatures observed. The thickness $t$ of this interface starts to increase nonlinearly with temperature above $206 \mathrm{~K}$ (Figure 3). Our experiments were conducted over two neutron-beam periods. In each run, one sample of each particle diameter was investigated. Irrespective of particle diameter, the temperature trend in $t$ was similar between the two runs but more pronounced in the first run, leading to relatively large uncertainties on the average $t$, but not affecting the conclusions about the temperature ranges over which changes occur.
The increase in $t$ (Figure 3 ) indicates that the outermost water molecules become more mobile and more disordered than the bulk material. This phenomenon is well known from hail, ice, and snow physics, where it is often referred to as surface pre-melting or quasi-liquid layers. It is usually attributed to a reduction of the free surface energy of the ice by this reorganization of molecules (Dash et al. 1995; Li \& Somorjai 2007, and references therein), although the names are misleading, as the surface is not truly liquid in such cases.

The observed temperature dependencies suggest that the $\AA$ A-scale surface properties of icy particles are impacting on collision experiments. Below $\approx 210 \mathrm{~K}$ collisional outcomes are temperature independent (Bridges et al. 1996; Gundlach \& Blum 2015; Hill et al. 2015), and this equates to the regime where the thickness of the diffuse surface layer is invariant. Above $\approx 210 \mathrm{~K}$, the thickness of this layer increases (nonlinearly with temperature) and temperature dependence is observed in collisional outcomes (Gundlach \& Blum 2015).

Typical collisional studies would use $\mathrm{H}_{2} \mathrm{O}$ samples, while our neutron scattering characterization required the use of $\mathrm{D}_{2} \mathrm{O}$. In general, the structural differences between the two materials are at a level of $<4 \%$ in terms of the intramolecular bond length and even smaller on intermolecular scales (Soper \& Benmore 2008). Isotope effects on molecular mobility are very complex, but overall the heavier $\mathrm{D}_{2} \mathrm{O}$ molecules $(20 \mathrm{amu})$ are less mobile than $\mathrm{H}_{2} \mathrm{O}$ ones $(18 \mathrm{amu})$. For example, at $298 \mathrm{~K}$ the diffusion coefficients are $(2.109 \pm 0.003) \times 10^{-5} \mathrm{~cm}^{2} \mathrm{~s}^{-1}\left(\mathrm{D}_{2} \mathrm{O}\right)$ and $(2.272 \pm 0.003) \times 10^{-5} \mathrm{~cm}^{2} \mathrm{~s}^{-1}\left(\mathrm{H}_{2} \mathrm{O}\right)$ (Eisenberg \& Kauzmann 2005); the triple point of $\mathrm{D}_{2} \mathrm{O}$ is slightly higher (276.967 K) (Markó et al. 1989) than that of $\mathrm{H}_{2} \mathrm{O}(273.16 \mathrm{~K})$. Therefore, $\mathrm{H}_{2} \mathrm{O}$ samples can be assumed to show slightly thicker diffuse interface layers than observed in this work, although the differences are likely to be at a level of a few percent only.

\subsection{Are the Icy Particles in Laboratory Collision Experiments Good Analogs?}

Interstellar ices are either formed by vapor deposition of gasphase water onto cooling dust grains (e.g., Visser et al. 2009) or through the chemical vapor deposition of $\mathrm{H}$ and $\mathrm{O}$ atoms to eventually form water ice (e.g., Cuppen et al. 2010; Accolla et al. 2013). Either way, the subsequent thermal or energetic processing of such ices results in crystallization of the material (e.g., Baragiola 2003; Burke \& Brown 2010), so that both amorphous and crystalline ices have been detected in accretion disks around young stars (Schegerer \& Wolf 2010; Terada \& Tokunaga 2012; Boogert et al. 2015).

All ice collisional experiments to date (e.g., Bridges et al. 1984; Deckers \& Teiser 2016), including ours (e.g., Gundlach \& Blum 2015; Hill et al. 2015), must have been colliding crystalline ice particles, as no method has yet been reported to effectively produce amorphous particles suitable for collision experiments.

From this work we can now confirm that the trends observed in collision outcomes as a function of temperature cannot be attributed to changes in ice-phase composition of crystalline ice. Therefore, under suitable $P-T$ conditions all crystalline ice analogs, be they $I_{c}, I_{h}$, or a mixture of both, are well suited to replicate grain collisions in astrophysical environments, where icy grains are dominated by crystalline icy material, e.g., heated regions of PPD or ring systems and planetary moons and atmospheres. It remains an open question (but beyond the 
a
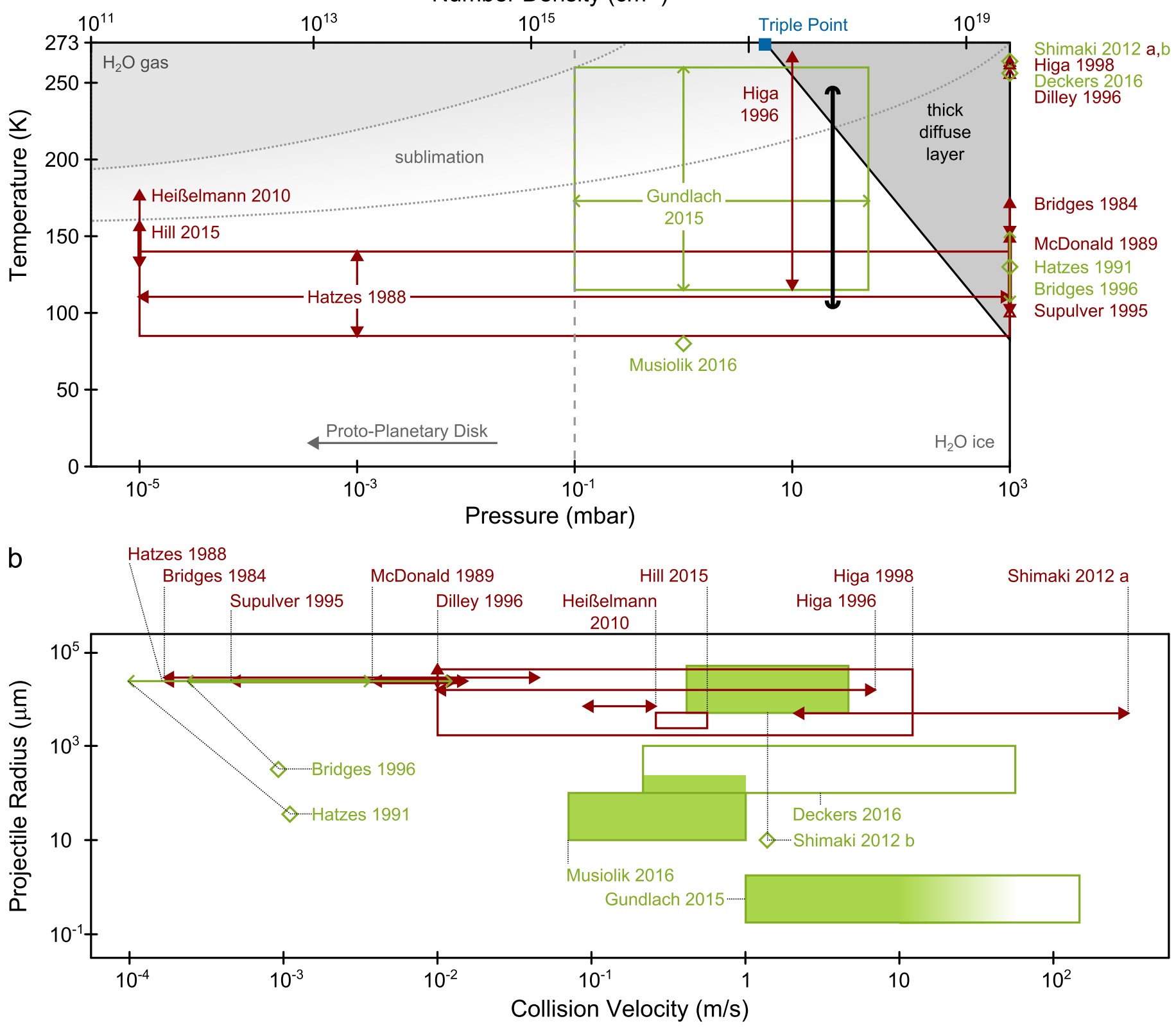

Figure 5. (a) Schematic summarizing the $P-T$ conditions or ranges of previous collision experiments (red and green symbols/lines), together with those under which ice can exist (white area) in the laboratory and in protoplanetary disks (PPDs). The region in which sublimation occurs will depend on pressure and composition of the ambient gas (more precisely on the partial $\mathrm{H}_{2} \mathrm{O}$ pressure), as depicted by the dotted lines. In PPDs, the ice pressure regime is limited by the snowline (dashed line: snowline pressure for a typical PPD, see Cieza et al. 2016, and references therein). Thin diffuse surface layers were observed across the whole temperature range of this work, above a critical temperature these start to thicken as the ice is warmed and strongly impact collisional outcomes. This critical temperature will depend on the ambient pressure as (qualitatively) depicted by the dark gray area. (b) Schematic summarizing the velocities, $v$, and radii, $r$, used in the above collision experiments. Partially sticking experiments are labeled within the schematic (green), and nonsticking experiments are labeled above the schematic (red). The ranges across which partial sticking was observed are shaded (green), the shading gradient on Gundlach (2015) (Gundlach \& Blum 2015) indicates the observed $T$-variance. For those studies where centimeter-sized projectiles featured micrometer-sized structures the mean size of these substructures is shown (diamonds) and connected to the experiment ranges via dotted lines. 
scope of this paper), whether collisional outcomes are the same for entirely amorphous icy particles or particles dominated by amorphous ice.

The key to icy particle collisional behavior, at least in laboratory experiments (e.g., Heißelmann et al. 2010; Gundlach \& Blum 2015; Hill et al. 2015), must be the diffuse surface layer, which increases the water-ice stickiness through surface premelting. This effect is known to be promoted by any type of irregularity at the surface, such as polycrystallinity, surface contact, or impurities. As our samples show stacking disorder and are granular, we would expect thicker diffuse surface layers in comparison to carefully prepared flat single crystal ice samples (e.g., Dosch et al. 1995), where it has previously been demonstrated that surface pre-melting can even occur at pressures as low as $P=6 \times 10^{-3}$ mbar, which is well within the pressure regime expected for a typical protoplanetary disk (Figure 5(a)).

Figure 5 summarizes the $P-T$ regimes for our, and other, planet-formation studies, putting them in context with the conditions typically found in protoplanetary disks. While our neutron scattering characterization was necessarily done at a constant pressure (30 mbar $\mathrm{He}$ ), given the experimental constraints we must assume that the critical temperature at which the thickness of the diffuse surface layer increases upon heating will change with pressure and composition of the ambient gas. This is qualitatively depicted by the dark gray area (Figure 5), whose border connects our observation at $30 \mathrm{mbar}$ to $\mathrm{H}_{2} \mathrm{O}$ 's triple point. The atmospheric pressure end of the curve is informed by earlier observations that $\mathrm{H}_{2} \mathrm{O}$ molecules become mobile enough to restructure the surface and reduce the number of incompletely coordinated molecules at the boundary at temperatures as low as 60-120 K (Devlin 2001).

Most importantly, Figure 5(a) shows that all collision studies that resulted in a certain percentage of sticking were indeed carried out under $P-T$ conditions where a diffuse surface layer exists on the icy particles; and temperature dependence in the collisional outcomes is induced where the thickness of that layer starts to increase. The one caveat to this is the work of Musiolik et al. (2016), whose experiments were conducted under $P-T$ conditions far from those where the diffuse surface layer dominates sticking outcomes. However, their experimental conditions relied on equilibria between gas, liquid, and solid water, which would result in dynamic exchange of water molecules at the particle surfaces, in a so-called dynamic liquid-like surface layer, as also reported in atmospheric studies of icy grains (Dash et al. 1995; Li \& Somorjai 2007, and references therein). Nevertheless, as Figure 5(a) shows, care should be taken when considering the outcomes of ice collision studies, as the influence of the $P-T$ conditions on the surface structure and behavior of the particles seems, from this work, to be at least as important as the velocity or size of the particles. The open question remains to what extent the diffuse surface layer impacts collision outcomes under the $P-T$ conditions in a protoplanetary disk.

\section{Conclusions}

We have characterized the ice-particle analogs used in our laboratory collision experiments (Gundlach \& Blum 2015), exploiting neutron scattering and cryo-SEM to determine whether they are good analogs for protoplanetary disk environments, whether their ice phase affects the collisional outcome, and whether their surface structure plays a dominant role in collisions.

Our analysis shows that neither changes in specific surface area nor in crystalline ice phase tie-in with previously observed temperature dependencies of collisional outcomes. The key to these temperature effects must be the increasing thickness of the diffuse surface layer, which at 30 mbar pressure is present across all investigated temperatures (103-247 K), but starts to increase in thickness above $\approx 210 \mathrm{~K}$, matching the observed onset of temperature dependent collision outcomes.

Ideally, experiments would always be performed at $P-T$ conditions that are expected for protoplanetary disks. Where that is prevented by the experimental procedures, care should be taken to avoid $T$-dependent outcomes induced by the diffuse surface layer. Therefore, at pressures of a few mbar, collision experiments should be performed below $210 \mathrm{~K}$, as changing surface structural properties of the ice will otherwise affect the collision outcomes.

While the typical production methods for crystalline ice analogs do not impact on the collision outcomes, the collision environment does. Therefore, in laboratory studies of icy particle collisions the parameter space, particularly with reference to $P$ and $T$, must be clearly defined and controlled. In collisional studies to date significant parameter space w.r.t pressure, temperature, size, and velocity is yet unexplored (Figure 5) and particle sticking has so far only been observed at pressures higher than expected in protoplanetary disks. Unlike silicon-based dust particles, bulk and surface structures of ice are influenced by the surrounding $P-T$ conditions. Nevertheless, planet formation models currently prioritize parameters such as particle velocity and size. Further investigation of the $P-T$ range at which diffuse surface layers affect collision outcomes is clearly warranted, to enable such findings to be incorporated into future models.

We kindly acknowledge helpful discussions with Richard Heenan (ISIS Facility). Experiments at the ISIS Pulsed Neutron and Muon Source were supported by a beamtime allocation on the near and intermediate range order diffractometer NIMROD from the Science and Technology Facilities Council, RB1520425. S.G. and H.J.F. acknowledge support from The Open University. Astrochemistry at the Open University is supported by STFC under grant agreements No. ST/M007790/ 1, ST/M003051/1, ST/N006488/1, ST/N005775/1, and ST/ L000776/1 as well as Royal Society International Exchange Award (IE/14/3). S.G. and H.J.F. both gratefully acknowledge travel support associated with this project from the EU COST Action CM1401 Our Astrochemical History, including STSM100615-061929 linked with this work. B.G., J.R., and J.B. acknowledge support from TU Braunschweig and DLR under grant 50WM1536.

Software: GudrunN (Soper 2011, 2013).

\section{ORCID iDs}

S. Gärtner (i) https://orcid.org/0000-0002-0786-5475

T. F. Headen (i) https://orcid.org/0000-0003-0095-5731

T. G. A. Youngs (iD https://orcid.org/0000-0003-3538-5572

D. T. Bowron (ib https://orcid.org/0000-0002-4557-1929

J. Blum (i) https://orcid.org/0000-0003-1531-737X

H. J. Fraser (i) https://orcid.org/0000-0003-0972-1595 


\section{References}

Accolla, M., Congiu, E., Manico, G., et al. 2013, MNRAS, 429, 3200 Aumatell, G., \& Wurm, G. 2011, MNRAS, 418, L1

Baragiola, R. A. 2003, P\&SS, 51, 953

Blum, J., \& Wurm, G. 2008, ARA\&A, 46, 21

Boogert, A. A., Gerakines, P. A., \& Whittet, D. C. 2015, ARA\&A, 53, 541

Bowron, D., Soper, A., Jones, K., et al. 2010, RScI, 81, 033905

Bridges, F. G., Hatzes, A., \& Lin, D. 1984, Natur, 309, 333

Bridges, F. G., Supulver, K. D., Lin, D., Knight, R., \& Zafra, M. 1996, Icar, 123,422

Burke, D. J., \& Brown, W. A. 2010, PCCP, 12, 5947

Cieza, L. A., Casassus, S., Tobin, J., et al. 2016, Natur, 535, 258

Cuppen, H., Ioppolo, S., Romanzin, C., \& Linnartz, H. 2010, PCCP, 12, 12077

Dash, J., Fu, H., \& Wettlaufer, J. 1995, RPPh, 58, 115

Deckers, J., \& Teiser, J. 2016, MNRAS, 456, 4328

Devlin, J. P. 2001, JGRE, 106, 33333

Dilley, J., \& Crawford, D. 1996, JGRE, 101, 9267

Dosch, H., Lied, A., \& Bilgram, J. 1995, SurSc, 327, 145

Eisenberg, D., \& Kauzmann, W. 2005, The Structure and Properties of Water (Oxford: Oxford Univ. Press)

Feigin, L. A., Svergun, D. I., \& Taylor, G. W. 1987, in General Principles of Small-Angle Diffraction, ed. G. W. Taylor (Boston, MA: Springer), 25

Garaud, P., Meru, F., Galvagni, M., \& Olczak, C. 2013, ApJ, 764, 146

Gundlach, B., \& Blum, J. 2015, ApJ, 798, 34

Gundlach, B., Kilias, S., Beitz, E., \& Blum, J. 2011, Icar, 214, 717

Hatzes, A., Bridges, F., Lin, D., \& Sachtjen, S. 1991, Icar, 89, 113

Hatzes, A. P., Bridges, F. G., \& Lin, D. 1988, MNRAS, 231, 1091

Heißelmann, D. 2015, PhD thesis, Technische Universität zu Braunschweig

Heißelmann, D., Blum, J., Fraser, H. J., \& Wolling, K. 2010, Icar, 206, 424

Higa, M., Arakawa, M., \& Maeno, N. 1996, P\&SS, 44, 917

Higa, M., Arakawa, M., \& Maeno, N. 1998, Icar, 133, 310

Hill, C. R., Heißelmann, D., Blum, J., \& Fraser, H. J. 2015, A\&A, 573, A49

Hill, C. R., Mitterdorfer, C., Youngs, T. G., et al. 2016, PRL, 116, 215501 Hondoh, T. 2015, PMag, 95, 3590

Jost, B., Gundlach, B., Pommerol, A., et al. 2013, Icar, 225, 352

Kataoka, A., Tanaka, H., Okuzumi, S., \& Wada, K. 2013, A\&A, 557, L4

Kuhs, W. F., Sippel, C., Falenty, A., \& Hansen, T. C. 2012, PNAS, 109, 21259
Li, Y., \& Somorjai, G. A. 2007, J. Phys. Chem. C, 111, 9631

Malkin, T. L., Murray, B. J., Brukhno, A. V., Anwar, J., \& Salzmann, C. G. 2012, PNAS, 109, 1041

Malkin, T. L., Murray, B. J., Salzmann, C. G., et al. 2015, PCCP, 17, 60

Markó, L., Jákli, G., \& Jancsó, G. 1989, J. Chem., 21, 437

McDonald, J., Hatzes, A., Bridges, F., \& Lin, D. 1989, Icar, 82, 167

Mitterdorfer, C., Bauer, M., Youngs, T. G., et al. 2014, PCCP, 16, 16013

Musiolik, G., Teiser, J., Jankowski, T., \& Wurm, G. 2016, ApJ, 827, 63

Petrenko, V. F., \& Whitworth, R. W. 1999, Physics of Ice (Oxford: Oxford Univ. Press)

Ros, K., \& Johansen, A. 2013, A\&A, 552, A137

Schegerer, A. A., \& Wolf, S. 2010, A\&A, 517, A87

Sears, V. F. 1992, Neutron News, 3, 26

Shimaki, Y., \& Arakawa, M. 2012a, Icar, 218, 737

Shimaki, Y., \& Arakawa, M. 2012b, Icar, 221, 310

Sinha, S. K., Sirota, E. B., Garoff, S., \& Stanley, H. B. 1988, PhRvB, 38, 2297

Sirono, S.-I. 2011a, ApJL, 733, L41

Sirono, S.-I. 2011b, ApJ, 735, 131

Sirono, S.-I., \& Ueno, H. 2017, ApJ, 841, 36

Soper, A., \& Benmore, C. 2008, PhRvL, 101, 065502

Soper, A. K. 2011, GudrunN and GudrunX: Programs for Correcting Raw Neutron and X-ray Diffraction Data to Differential Scattering Cross Section, Technical Report RAL-TR-2011-013 (Swindon: STFC)

Soper, A. K. 2013, ISRN Phys. Chem., 2013, 279463

Strey, R., Winkler, J., \& Magid, L. 1991, JPhCh, 95, 7502

Su, T. J., Lu, J. R., Cui, Z. F., Thomas, R. K., \& Heenan, R. K. 1998, Langm, 14,5517

Supulver, K. D., Bridges, F. G., \& Lin, D. 1995, Icar, 113, 188

Terada, H., \& Tokunaga, A. T. 2012, ApJ, 753, 19

Testi, L., Birnstiel, T., Ricci, L., et al. 2014, in Protostars and Planets VI, ed. $\mathrm{H}$ Beuther et al. (Tucson, AZ: Univ. Arizona Press), 339

Visser, R., Van Dishoeck, E., Doty, S., \& Dullemond, C. 2009, A\&A, 495, 881

Wada, K., Tanaka, H., Suyama, T., Kimura, H., \& Yamamoto, T. 2008, ApJ, 677, 1296

Wang, H., Bell, R. C., Iedema, M. J., Tsekouras, A. A., \& Cowin, J. P. 2005, ApJ, 620, 1027

Woitke, P. 2015, EPJ Web Conf., 102, 00011

Woitke, P., Min, M., Pinte, C., et al. 2016, A\&A, 586, A103 